\title{
El problema de Dios en la fenomenologí a de Husserl. Angela Ales Bello, Edmund Husserl. Pensar a Dios, creer en Dios. Buenos Aires: Editorial Biblos, 2016.
}

\author{
Rubén Sánchez Muñoz \\ Universidad Popular Autónoma del Estado de Puebla \\ ruben.sanchez.munoz@upaep.mx
}

Contrario a lo que se puede pensar y creer respecto del lugar o no lugar que ocupa el problema de Dios en la fenomenología de Edmund Husserl, esto es, la idea de que este problema a Husserl no le interesaba, o que Husserl hizo epojé del tema de Dios en I deas I y luego se olvidó de retomarlo, así la labor de Ángela Ales Bello ha sido la de mostrar que el filósofo moravo se interesó profundamente en la cuestión de Dios y que es un tema fundamental dentro de la fenomenología de Husserl. En efecto, la filósofa italiana ha dedicado una parte importante de su trayectoria profesional a explorar el problema de Dios en la fenomenología de Edmund Husserl. En 1985 publicó Husserl. Sul problema di Dio y tres décadas después la obra que ahora comentamos. Por ello, podemos decir que en el trabajo de la autora vemos cumplirse la idea misma que Husserl tenía de la fenomenología como Arbeitsphilosophie, esto es como una filosofía de trabajo, donde los problemas se esclarecen en la práctica concreta, en la constancia misma del trabajo y el esfuerzo. El presente trabajo es un comentario crítico a la obra de la filósofa italiana Edmund Husserl. Pensar a Dios, creer en Dios.

Husserl mismo era consciente de las dificultades que podían tenerse a la hora de intentar comprender la fenomenología y a la hora misma de querer hacer análisis intencionales desde un enfoque fenomenológico. Ello ciertamente le generó una serie de incomprensiones y malentendidos. Estas dificultades las tenían ya sus alumnos directos, como Stein, Landgrebe, Heidegger y Fink, los integrantes del círculo de Gotinga y con mucha más razón quienes no trabajaban directamente con el fundador de la fenomenología. En la actualidad, con la publicación de sus obras completas, de la edición Husserliana, todavía seguimos cuestio- 
nándonos si hemos ganado con el tiempo mayor claridad sobre lo que es la fenomenología y si hemos conseguido avanzar en la conformación de una comunidad de fenomenólogos unidos por las mismas metas y los mismos fines.

El libro de Ales Bello consta de cuatro densos capítulos. Los traductores hacen una presentación de la obra y la autora una breve y lúcida introducción. En ella podemos ver la trascendencia del tema de estudio y el vínculo generativo que tiene este tema en la filósofa. Ella reconoce que abordar el problema de Dios desde la fenomenología es un verdadero "desafío". Si el problema de Dios es un tema marginal en la obra de Husserl, entonces ¿por qué leer los "análisis fenomenológicos de Husserl en clave teológica"? Ales Bello reconoce que el tema le interesa desde el punto de vista teórico, en primer lugar y, en segundo, le interesa las contribuciones que la fenomenología puede hacer a las ciencias humanas, es decir, a la historia, a la antropología y "a las ciencias" (p. 13). En este orden considera valioso preguntarse por la contribución de la fenomenología al tema de Dios.

A este desafío que representa el tema mismo del estudio, hay que sumarle dos cosas. 1. Que los análisis de Husserl son "difíciles” y 2. Que, por ello mismo, son lentos y, por tanto, para llegar a algún resultado es preciso hacer un "largo y tortuoso recorrido" (p. 15). La imagen que nos presenta Ales Bello de Husserl es la de un filósofo serio y comprometido, "que no quiere exponer rápido la opinión propia" y por ello "quiere seguir las vías transitables de hecho por parte del ser humano en su investigación y en su actitud teórica y práctica, con la esperanza de desvelar el sentido de la realidad" (p. 15). La autora, sin embargo, se esfuerza por hacer una presentación digamos inteligible de la fenomenología, recurriendo lo menos posible a términos técnicos, pero reconociendo la necesidad de hacerlo para ganar profundidad.

Así pues, el primer capítulo representa una valiosa introducción al pensar fenomenológico y lo intitula justamente "La fenomenología como filosofía sui generis". Aquí Ales Bello expone de un modo pedagógico - bajo el entendido de una buena pedagogía- algunos conceptos básicos de la fenomenología. Ello constituye un gran reto si revisamos el gran número de introducciones a la fenomenología de las que disponemos actualmente. El valor de la presentación de Ales Bello está no solo en la claridad expositiva, sino también en no detenerse a discutir este asunto y distraerse en ello. Va, en un sentido que ya es fenomenológico, a las cosas mismas. El objetivo de la fenomenología es "captar el sentido 
de la realidad", cayendo en la cuenta de que eso que llamamos real, en cualquiera de sus sentidos, es una realidad dada. La primacía del conocimiento en la descripción fenomenológica encuentra su justificación en que el conocimiento se convierte en el instrumento que nos permite "comprender cómo están hechas las cosas" ( p. 20). Pero el conocimiento está a su vez fundado en las operaciones de la subjetividad. Husserl se da cuenta que en las matemáticas $-\mathrm{y}$ en cualquier otra rama del saber - es preciso "retroceder a las operaciones que lo constituyen". El terreno o región donde se constituyen las distintas realidades que aparecen, ese es el terreno de la fenomenología, la nueva esfera de ser. Así que, a la altura de 1907 Husserl ya tiene una determinada armazón conceptual que le permiten hablar de la fenomenología entendida como “una reflexión-descripción de los fenómenos que se presentan a la subjetividad humana, y por sobre todo, de los fenómenos constituidos por los actos de conciencia" (p. 21). A juicio de Ales Bello, Husserl combina en sus descripciones dos tipos de investigación: "la del arqueólogo y la del explorador" (p. 21).

En efecto, Husserl ensayó varias vías de acceso a la conciencia a través de las vías de la reducción. La reducción - que es uno de los conceptos fundamentales de la fenomenología y uno también de los más problemáticos- tiene el siguiente sentido según Ales Bello: “el término reducción tiene el sentido de lo que es superfluo para alcanzar, precisamente, la meta. Y mientras se recorre el camino muchas cosas se ponen a un lado, la eliminación no es destrucción, sino un apartamiento y una no utilización" (p. 22). Husserl tiene la intención de devolverle a la filosofía su carácter científico, pero para hacerlo debe poner entre paréntesis el sentido de lo "científico" entendido desde un enfoque positivista. Por ello la intuición ocupa un lugar importante, en especial como intuición eidética, porque las ideas o esencias de las que habla la fenomenología no son ideas o esencias en sentido metafísico sino "en sentido lógico-gnoseológico" (p. 23). Por esta razón, en el principio de todos los principios, Husserl invita a atenernos a lo dado en la intuición y nada más. La epojé tiene como propósito justamente no dar por hecho la existencia del mundo espacio-temporal, sino más bien atenernos a lo dado en la experiencia vivida o en los "actos vividos conscientemente a fin de captar su sentido" ( p. 25). El objetivo de la reducción sería pues, acceder al terreno de la subjetividad trascendental, para la cual el filósofo moravo ensayó varias vías. Lo importante de las vías de la reducción para acceder a la subjetividad trascendental es que cada una de ellas "desemboca en una serie de 
observaciones en torno al problema "metafísico"... que se refiere a la realidad última, la divina" (p. 29).

Husserl asume que para cada objeto constituido existe una serie o tipología de actos de conciencia en los cuales el objeto se constituye, por ello, el estudio del problema de Dios debe considerar el método para llegar a las vivencias y luego "entender cómo se configura esa realidad" (p. 29). Se debe considerar además que si bien "el ser como realidad y el ser como conciencia son correlatos... son distintos".

De especial importancia resultan las reflexiones de Ales Bello sobre el valor de la fenomenología para las ciencias humanas, en especial para la antropología. Sobre este asunto la autora esclarece algunos de los principales problemas de la compleja relación entre fenomenología y antropología al final del primer capítulo.

El segundo capítulo lo dedica Ales Bello al análisis de "La cuestión de Dios como cuestión filosófica". En él explora varias vías que conducen hacia Dios, tales como la vía objetiva, la vía subjetiva, la vía intersubjetiva, la vía hacia Dios a través de la hylética, así como la teleología y la ética. Ales Bello muestra cómo en el filósofo moravo se hallan contenidos los grandes temas y problemas de la metafísica occidental, a saber: yo, mundo y Dios (p. 48). Desde la vía objetiva, Ales Bello subraya la importancia de la teleología y lo importante que es para Husserl el hecho de que el mundo "está ordenado" según una racionalidad que "demuestra poseer un fin" (pp. 48 s). La respuesta de Husserl, y la cual recupera la autora, es que "el pasaje a la conciencia pura, efectuada con el método trascendental, conduce inevitablemente a preguntarse, más bien, cuál es el fundamento de la actualidad que brota ahora de la correspondiente conciencia constitutiva" (cit. p. 49). Husserl busca el fundamento racional de esta actualidad y ello lo conduce a Dios entendido como trascendencia, una trascendencia que es distinta de la trascendencia del mundo. Ahora bien, la vía subjetiva que lleva a Dios y que está presente en Husserl, puede encontrarse en la historia de la filosofía en autores como Aristóteles, Tomás de Aquino, Descartes, Leibniz o Kant (p. 50). La vía subjetiva hacia Dios profundiza en el principio teleológico desde el interior de la vida de conciencia. Husserl busca en la conciencia esos "otros modos de anunciarse de la trascendencia" (p. 52). Para describir esta experiencia sui generis Husserl "exhorta a concentrarse en los actos de conciencia vividos" ( p. 54). Cabe decir que en este apartado Ales Bello realiza una comparación entre 
Husserl y san Anselmo que posee dentro del trabajo, a nuestro juicio, un alto valor.

Pues bien, la tercera vía hacia Dios es la vía intersubjetiva. Aquí la autora muestra lo importante que es la "constatación del cuerpo extraño a partir del cuerpo ajeno" y el importante lugar que juega en ello la empatía. Lo que tenemos es el descubrimiento de una comunidad intersubjetiva de mónadas que de alguna manera se encuentran entrelazadas. La meditación sobre la conciencia extraña conduce a Husserl a "la conciencia omnicomprensiva divina" o a la cuestión por la "mónada suprema" (p. 64). Dios sería pensado por Husserl en el sentido de una "conciencia que comprende en sí todo, como conciencia de todo" o también como "conciencia omnienglobante" (pp. 66 s.). Así, en palabras de la autora, "el cotejo con Dios es interesante, pues indica, en primer lugar, que el ser humano conoce los límites experienciales propios porque tiene la capacidad de confrontarse con una realidad perfecta que lo supera" (p. 68). En este tenor, Ales Bello recurre a la noción de mónada que Husserl toma prestada de Leibniz y muestra el paso de la mónada a la Mónada Suprema. A diferencia de Leibniz, Husserl considera que las mónadas tienen ventanas y, de este modo, en los años 20's la intersubjetividad en Husserl se convierte en intermonacidad (p. 69). El trabajo de Ales Bello nos introduce en esta relación estrecha que hay entre Husserl y Leibniz y sienta bases sólidas de un estudio comparativo entre ambos. Pues bien, el problema de Dios en Husserl tiene un vínculo directo con la metafísica. Así como Dios, otros temas tales como el nacimiento, la muerte, el sueño sin sueños, el inconsciente y la muerte, son problemas límite. La cercanía entre Husserl y Leibniz o si se prefiere los paralelismos entre ambos se deja ver de manera muy profunda en estas cuestiones. Husserl parece aceptar, al igual que Leibniz, que toda la realidad está constituida por mónadas que se encuentran en "niveles diferentes". En el aparecer y desaparecer de las mónadas lo que tendríamos es una "fuerza originaria" que, a decir de Husserl, permite un "desarrollo ascendente". Se trata de un desarrollo que tiene como fin o telos "la razón y la forma de la perfección" (p. 78). Para Husserl, la racionalidad es "la capacidad de prefijarse metas válidas y de perseguirlas en la correlación entre el momento teórico que las pone en evidencia y el momento práctico que las actualiza" ( p. 78).

Una cuarta vía que conduce a Dios es la hylética. Sobresale en esta línea el lugar importante que ocupa el cuerpo (Leib). Lo relevante del cuerpo viviente como contenido hylético o material, está en que a pesar de que "el universo 
hylético es un universo no egológico", en él se da un nexo con las afecciones. En los sentimientos sensibles, en las sensaciones de placer y dolor, bienestar o malestar corporal, en todo "el yo está siempre presente como el lugar de las afecciones y siempre está activo" ( p. 81). La conciencia, pues, está ligada a su base hylética. En este nexo puede rastrease un sentido teleológico. Comentemos brevemente cómo se da esto. Dice Ales Bello que "la hyle es la materia a la cual la nóesis da un significado, y se torna así materia para ulteriores operaciones, por ejemplo: el agrado que produce la visión de un color me impulsa a elegir ese color, a valorarlo positivamente y así se torna "materia" de un juicio" (p. 89). Husserl se encuentra ante el hecho de que en el mundo "nada es casual" y que "es necesario rastrear desde las dimensiones más profundas una "teleología", una finalidad, y por ende la remisión a una "facticidad originaria" puede comprenderse hasta el fondo si se constata que ella tiene su fundamento en Dios" (p. 92).

La última vía que Ales Bello expone es la vía ética. En ella la finalidad adquiere un valor moral. La reflexión sobre los valores adquiere sentido en esta vía, porque están en relación con la subjetividad humana y, en especial, con la vida en comunidad de las personas. La comunidad, como forma de asociación humana, ocupa un lugar central dentro de la fenomenología de Husserl. El filósofo confía en que "la humanidad llegaría a madurar éticamente mediante normas ideales". El despliegue de la razón en la historia alcanza un sentido práctico que involucra a la ética y a la cultura cuando es formulada desde la vida comunitaria. La ética de Husserl tiene en cuenta el lugar que ocupa la vida afectiva de las personas en sus relaciones interpersonales y con el mundo circundante. Tiene en consideración el amor, porque este sentimiento “determina para quien lo siente un deber respecto de la persona amada" (p. 94). En el amor se anuncia una llamada interior hacia un deber absoluto. Este deber absoluto "está enlazado con la acción de valorar" (p. 95). La presencia de fines en el mundo y la libertad del hombre para realizarlos anuncia que "un Dios gobierna el mundo".

La segunda parte del libro trata del tema "Creer en Dios" y lo conforman los capítulos III y IV. El capítulo III es una “Aproximación fenomenológica al tema de la religión" y en él, Ales Bello rastrea aquellos pasajes de la obra de Husserl en los cuales el filósofo moravo se refiere al modo de anunciarse de las trascendencias y del sentido de la conciencia religiosa. Desde el punto de vista de la fenomenología, la donación originaria de la experiencia de Dios encuentra una 
fundamentación racional. "La conciencia sabe y es religiosa en el sentido de que tal conciencia es la conciencia de la presencia de Dios y a la vez de su no reductibilidad a la conciencia misma, por ello su presentarse como "Otro" se basa en el reconocimiento de su presencia-ausencia" ( p. 102). Ales Bello resalta tres aspectos de la experiencia religiosa: 1. "se da de manera inmediata e intuitiva"; 2. “es relativa a algo «potente» y «unitario»" y 3. "la reflexión teórica sigue y explicita el contenido de dicha experiencia" (p. 103). Del tercer aspecto se sigue que "el creer en Dios precede de hecho al pensar en Dios" (p. 103).

Al cuestionar la autora la posibilidad de una vía mística del conocimiento de Dios en Husserl, recurre a un texto de Herda Walther publicado en 1923. Se refiere a la fenomenología de la mística. Sobre este trabajo Walther recibió una carta de Husserl de siete páginas. Ales Bello se pregunta si en esa carta podemos encontrar vestigios sobre la postura de Husserl respecto de la experiencia mística. En todo caso su postura se enfoca más hacia el trabajo de la filósofa para sostener que, si bien Husserl no excluye la experiencia mística como posibilidad, “no está dispuesto a incluirla en sus investigaciones" (p. 107).

Investigaciones fenomenológicas recientes sobre ética y cultura han puesto en evidencia la importancia que tenía para Husserl la figura de Cristo y la presencia del cristianismo en la historia de la humanidad. En este capítulo Ales Bello dedica un profundo análisis a los temas de la conversión, el lugar del cristianismo en la historia de las religiones, Cristo como fuente de amor ético y, finalmente, las relaciones entre teología y fe. Nos recuerda que hacia 1882 Husserl se sintió atraído por la figura de Cristo tras la lectura del Nuevo Testamento y que entró en la comunidad cristiana luterana de Viena. Husserl centra su mirada en la relación que tiene la conversión en la vida de un hombre y la búsqueda de la verdad y se pregunta: “¿De qué modo satisface Cristo una exigencia de fe pero colma también la aspiración profunda de búsqueda intelectual de la verdad?" (p. 109).

Lo que impresiona a Husserl del cristianismo — de acuerdo con Ales Belloes su universalidad en comparación con otras religiones. Esta aspiración de universalidad la encuentra Husserl dentro de la civilización occidental tanto en la filosofía como en la religión cristiana. Al preguntarse Ales Bello por qué Husserl se convirtió al cristianismo, da un argumento que está en favor de la ética. La ética para Husserl "está fuertemente vinculada a valores y normas universales, pero estas deben ser elegidas y aceptadas libremente. Le parece que el cristianismo tiene en sí tal apertura contenida precisamente en el mensaje de Cristo" 
(p. 112). En efecto, la figura de Cristo aparece como fuente de amor ético, como el "prototipo del amor ético" y la expresión más alta del amor es el amor por el enemigo. El amor auténtico busca el bien para los otros sin excluir a los extraños y a los enemigos. Por ello Ales Bello enfatiza que, para Husserl, "el amor a Cristo supera la dicotomía amigo-enemigo, supera los vínculos étnicos, las afinidades espontáneas o las elecciones ideológicas, se expande hasta abarcar la humanidad entera" (p. 116). Muestra también el sentido de la teología natural o racional como vía no confesional hacia Dios, que es la vía que sigue Husserl y desde la cual emanan estas reflexiones. En su obra podemos constatar, en efecto, esta conexión que hay entre religión, ética y metafísica, las cuales vienen de la mano de esta búsqueda de un saber universal.

El capítulo final trata de "La religión como objeto de análisis fenomenológico". En él, Ales Bello muestra la concordancia que hay entre el método fenomenológico y la profundidad y amplitud que llega a tener el tema de la religión en la obra de Husserl. La fenomenología "excava en la interioridad humana" y además "examina las expresiones y configuraciones humanas de esa experiencia" ( $p$. 123). Sobre ello la autora resalta las cartas de Husserl con Erich Przywara y Rudolf Otto. Aunque Husserl se propone describir el fenómeno religioso desde el ámbito de la conciencia, excavando en las profundidades de la subjetividad humana, se topa aquí con las "cuestiones últimas" propias de la metafísica. Sostiene que "sólo comprendiendo la naturaleza de la conciencia trascendental se puede comprender la trascendencia de Dios" (p. 124). Así que, con la ayuda de la fenomenología los problemas ético-religiosos podrían esclarecer su sentido y validez de ser. Para mostrar lo que allí se afirma, Ales Bello realiza una breve pero profunda descripción del fenómeno religioso y presenta las líneas generales de una arqueología fenomenológica de lo sagrado.

En el primer punto, la autora resalta la importancia de la fenomenología de la religión de G. van der Leeuw al identificar un núcleo que es común a todas las religiones. Van der Leeuw busca las "características esenciales" del fenómeno religioso y encuentra un "núcleo fundamental de la existencia humana en la apertura religiosa" (p. 127). En su búsqueda de la potencia, el ser humano se encuentra con una Potencia "que colma la espera". Esta búsqueda conduce al encuentro con algo o Alguien "que es esencialmente extraño, o sea, que no depende de su deseo o de su intelecto como presencia evidente y, en consecuencia, veritativa, que en todo caso siente profundamente aún si no lo quiere ver y no lo 
quiere aceptar" y añade, allí mismo, que "Nos hallamos en la línea de la verticalidad" (p. 128). Ales Bello insiste que se trata de la experiencia religiosa quoad nos y afirma que lo sagrado "es una presencia cuyo núcleo es hylético" (p. 131). Por ser así, en la experiencia religiosa en su sentido hylético se puede ver la centralidad que ocupa el cuerpo. Ello es así debido a que "las vivencias elementales están ligadas a las estructuras cinestésicas, o sea, a los movimientos de la corporalidad y, en consecuencia, a tal punto connotadas por la hyle cinestésica, que todo es movimiento, acción" (p. 130).

En las últimas páginas, siguiendo a Husserl, Ales Bello habla de una “Arqueología fenomenológica". De acuerdo con ello, el trabajo de la fenomenología sería un trabajo de excavación. La pregunta retrospectiva que busca el sentido de algo conduciría a las fuentes últimas y por ello mismo a los Archai. Por esta razón, la fenomenología permite tener acceso a las experiencias originarias del fenómeno religioso. La arqueología fenomenológica se propondría excavar en las diversas manifestaciones culturales del fenómeno religioso en el mundo de la vida propio de las culturas, reduciendo el fenómeno a la "esfera de las vivencias" (p. 136).

Luego de ello, Ales Bello presenta un valioso ejercicio donde expone algunos ejemplos de excavación fenomenológica. Decimos que este ejercicio es valioso a razón de que la aplicación de la propuesta a casos concretos nos enseña a poner en práctica el método. En muchas investigaciones fenomenológicas -incluidas las del propio Husserl- se echa de menos los ejemplos concretos. Así que, la aproximación que hace la filósofa a la antropología cultural y a la historia de las mitologías, nos permite apreciar el valor de los informes de los antropólogos culturales y el análisis de los signos que aparecen en sus descripciones. En efecto, Ales Bello resalta la importancia de los trabajos de Lévy-Bruhl, Claude LévyStrauss y T.G.H Strehlow y abre líneas de trabajo hacia una arqueología fenomenológica que se extiende por los terrenos de la antropología cultural. 